\title{
A New Compact Octagonal Shape Perfect Metamaterial Absorber for Microwave Applications
}

\author{
Mohammad Jakir Hossain ${ }^{1, *}$, Mohammad Rashed Iqbal Faruque ${ }^{1, *}$ (D), \\ Mohammad Tariqul Islam 2,* (D) and Kamarulzaman bin Mat ${ }^{2, *}$ \\ 1 Space Science Centre (ANGKASA), Institute of Climate Change (IPI), Universiti Kebangsaan Malaysia, \\ Bangi 43600, Selangor, Malaysia \\ 2 Department of Electrical Electronic and Systems Engineering, Universiti Kebangsaan Malaysia, \\ Bangi 43600, Selangor, Malaysia \\ * Correspondence: jakir@siswa.ukm.edu.my (M.J.H.); rashed@ukm.edu.my (M.R.I.F.); \\ tariqul@ukm.edu.my (M.T.I.); kamarulzaman@ukm.edu.my (K.b.M.); Tel.: +60-1-0293-8061 (M.R.I.F.)
}

Received: 31 October 2017; Accepted: 29 November 2017; Published: 6 December 2017

\begin{abstract}
A new compact octagonal shape perfect metamaterial absorber (PMA) design, numerical simulation, fabrication, and investigational verification of unit cell that is based on a simple structure are presented in this paper. The suggested structure comprised of three layers, in which interact to produce the plasmonic resonances. The finite-integration technique (FIT) based Computer Simulation Technology (CST) microwave electromagnetic simulator was utilized to examine the design parameters and conduct absorption analysis. The design structure exhibited peak absorption values as $99.64 \%$ and $99.95 \%$ at frequencies $8.08 \mathrm{GHz}$ and $11.41 \mathrm{GHz}$, respectively. The absorption characteristics were analysed using the polarization angle of the structure, layer thickness, PMA with resistive load, and number of rings. An N5227A vector network analyser was used for the measurement. The measured results of the fabricated prototype were in good agreement with the simulation results. The suggested perfect absorber structure enables innumerable application aimed at X-band for applications like, defence, security, and stealth technology.
\end{abstract}

Keywords: compact; X-band applications; perfect metamaterial absorber; octagonal ring

\section{Introduction}

Artificial metamaterials (MMs) are engineered composites consisting of sub-wavelength metallic structures in a host dielectric medium, which are engineered to obtain unconventional properties that are not found naturally. Due to the unconventional electromagnetic properties of numerous metamaterials, namely the (-) ve permittivity, (-) ve permeability, (-) ve refractive index, and invisibility, the design and application of MMs has gained the priority of vigorous research [1,2]. Nevertheless, MMs are also being extensively studied for different applications, for instance, perfect absorbers through the wide electromagnetic spectrum from millimetre to nanometre wavelengths [3,4], multiband absorber [5], polarization insensitive absorber [6], imagers and detectors [7,8], and broad band absorber [9], smart antenna, and beam shaping devices [10,11]. A $24 \times 24 \mathrm{~mm}^{2}$ Jerusalem cross with meandered load absorber depicts absorptions of more than $95 \%$ at $14.75 \mathrm{GHz}$ and $16.1 \mathrm{GHz}$ [12]. Lin et al. recommended a metamaterial unit cell structure with $10.92 \times 10 \mathrm{~mm}^{2}$ dimension that was applicable in microwave regime. The absorption peak of the structure was $96.5 \%, 96.8 \%$, and $99.6 \%$ at $2.15 \mathrm{GHz}, 2.28 \mathrm{GHz}$, and $2.38 \mathrm{GHz}$, respectively [13]. Zhao et al. offered $10 \times 10 \mathrm{~mm}^{2}$ ultra broadband perfect absorber based on an electric split-ring resonator (ESRR) loaded with lumped resistors. The design structure displayed the absorption of $99.3 \%, 97.1 \%$, and $98.6 \%$ at $5.45 \mathrm{GHz}, 15.46 \mathrm{GHz}$, and $19.48 \mathrm{GHz}$, respectively [14]. Dincer et al. [15] suggested the design for an absorbing metamaterial element with a near unity absorbance. They designed, fabricated, characterized, and analysed a metamaterial absorber (MA) with an absorbance of around 
$99.99 \%$ at $5.48 \mathrm{GHz}$ and $99.92 \%$ at $0.865 \mathrm{THz}$. However, the dimension of the unit cell structure was $36 \times 36 \mathrm{~mm}^{2}$. Hossain et al. recommended a design structure of $12 \times 12 \mathrm{~mm}^{2}$ composite double negative metamaterial for multi-band operation and reported effective medium ratio was 7.44 [16]. On the other hand, the above author analysed double negative characteristics and compactness, but absorption properties were not analysed in their study. Islam et al. [17] proposed multi-band split S-shaped metamaterial structure for absorption analysis, whereas authors obtained maximum $55 \%$ absorption. Kim et al. proposed a dual-band multilayer metamaterial absorber in the megahertz region, which were absorptions of $96 \%$ in the $4.0-6.0 \mathrm{GHz}$ range because of the irregular thickness of the resistive sheets [18]. A multi-band perfect metamaterial absorber based on spiral showed the absorption of $99.4 \%, 96.7 \%$, and $99.1 \%$ at three resonant frequencies $9.86 \mathrm{GHz}, 12.24 \mathrm{GHz}$, and $15.34 \mathrm{GHz}$, simultaneously [5]. Wen et al. suggested a dual-band metamaterial absorber in the terahertz area, which was two discrete absorptions of $80.8 \%$ and $63.4 \%$ near 0.45 and $0.92 \mathrm{THz}$ [19]. A dual-band MA design, fabrication, and characterization was offered by Tao et al. [20]. Their MA exhibited absorption peaks of $85 \%$ and $94 \%$ at $1.4 \mathrm{THz}$ and $3.0 \mathrm{THz}$, respectively. Polarization-independent MAs proposed by Kollatou et al. that performed in the microwave regime. The maximum absorption value was $95.81 \%$ at $10.31 \mathrm{GHz}$ [21].

In this paper, compact octagonal shape perfect metamaterial absorber exhibits dual resonance at X-band. The absorption has been changed by altering the polarization angle of unit cell, thickness of substrate and metallic materials, number of rings, and resistive load. Moreover, the size of the proposed unit cell is $10 \times 10 \mathrm{~mm}^{2}$, which is physically smaller than the stated metamaterials unit cells in literature $[12,13,15,17,22-24]$. Moreover, the design structure provides high absorption peaks of $99.64 \%$ at $8.08 \mathrm{GHz}$ and $99.95 \%$ at $11.41 \mathrm{GHz}$, respectively. It is observed that the absorption of the proposed design is better than the suggested metamaterials unit cells in [12-14,17-25]. To determine the absorption parameters, the Computer Simulation Technology (CST) microwave studio suite simulator 2015 is used.

\section{Design, Numerical Simulation, and Experiment}

The proposed perfect metamaterial absorber (PMA) consists of three octagonal shape resonators on the dielectric material and a ground plane backside. The proposed perfect metamaterial absorber structure and structural parameters are shown in Figure 1. All of the metallic elements of the PMA are made of copper with a conductivity of $5.8 \times 10^{7} \mathrm{~S} / \mathrm{m}$ and the thickness of copper resonators are $35 \mu \mathrm{m}$ that are printed on a substrate with standard relative permittivity $\varepsilon=4.3$, as well as loss tangent $\delta=0.025$. The parameters of the structure are $\mathrm{L}_{\mathrm{s}}=10 \mathrm{~mm}, \mathrm{~W}_{\mathrm{s}}=10 \mathrm{~mm}, \mathrm{~W}_{1}=\mathrm{W}_{2}=\mathrm{W}_{3}=0.8 \mathrm{~mm}$. The double-sided copper laminated PCB (Printed Circuit Board) (Shenzhen Zhongxinhua Electronics Co., Ltd., Shenzhen, China) is available in the market. Using LPKF S63 PCB prototyping machine (LPKF Laser \& Electronics, Tualatin, OR, USA) or chemical process, it is easy to fabricate the design structure. Low cost, high efficient flame retardant4 (FR4) epoxy dielectric material (Shenzhen Zhongxinhua Electronics Co., Ltd., Shenzhen, China) was utilized as substrate material.

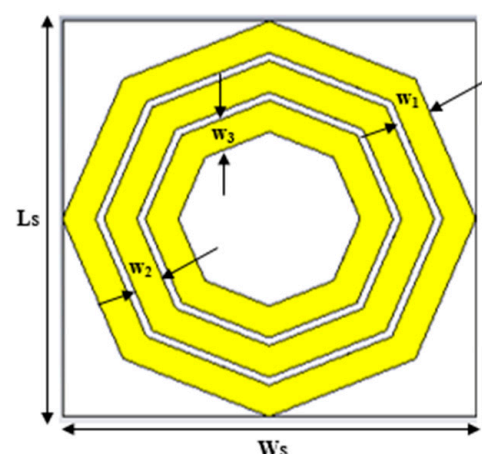

(a)

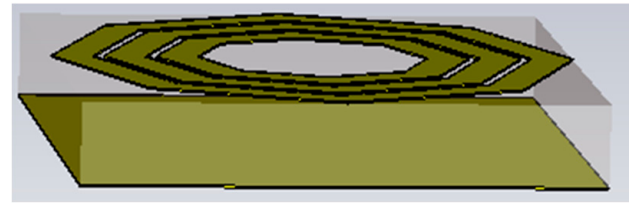

(d)

Figure 1. Cont. 


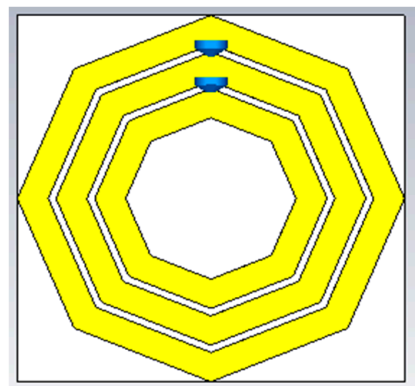

(b)

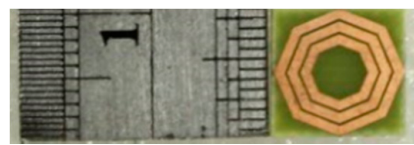

(c)

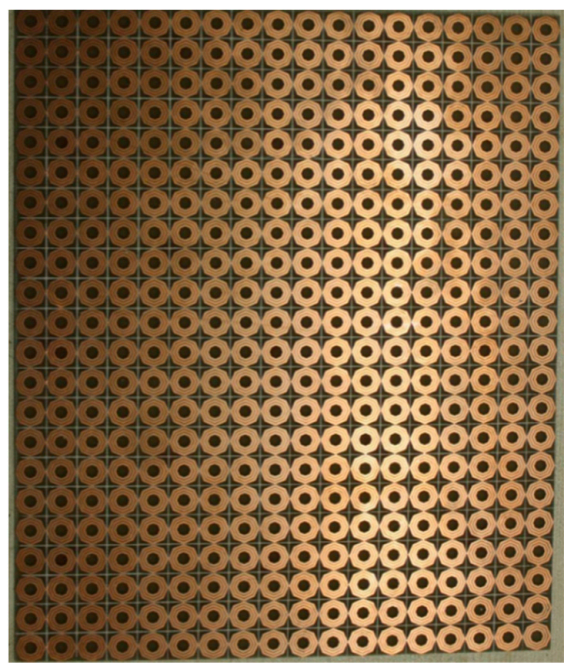

(e)

Figure 1. (a) The proposed sketch of perfect metamaterial absorber (PMA); (b) Unit cell with resistive load; (c) The fabricated unit cell structure; (d) Side view of PMA; and, (e) Fabricated array of PMA.

Finite-integration technique (FIT) based CST microwave studio suite simulator has been implemented to examine this design structure in this paper. The electric field and magnetic field have been polarized along the $\mathrm{x}$-axis and the $\mathrm{y}$-axis, respectively, thus wave propagation is along the $\mathrm{z}$-axis. The boundary conditions of perfect magnetic conductor (PEC) and the perfect electric conductor (PMC) are utilized along the $x$-axis and y-axis, individually, and two waveguide ports are placed on the positive and negative z-axis. In addition, the periodic boundary conditions with the waveguide ports are used in simulation. The schematic drawing of the proposed structure, unit cell with resistive load, fabricated unit cell, side view of the unit cell, and array of the structure are demonstrated in Figure 1.

CST based frequency domain solver was used to determine the reflection coefficient in simulation at 1001 frequency samples. The boundary condition and measurement set up are shown in Figure 2 . The fabricated sample encloses $18 \times 22$ arrays of the structure of copper materials, and the dimension of the fabricated sample are $180 \times 220 \mathrm{~mm}^{2}$. The measurement has been performed with two waveguides in the free-space environment. The scattering parameters have been measured by the PNA network analyser (N5227, Agilent Technologies Sdn. Bhd., Petaling Jaya, Malaysia) where the frequency range of the device was 10 MHz-67 GHz. In addition, a calibration kit (Agilent N4694-60001, Agilent Technologies Sdn. Bhd., Petaling Jaya, Malaysia) was utilized to calibrate the network analyser. For that reason, the measurements were completed precisely. The absorption of the design structure is determined using the following equation, $A(\omega)=1-R(\omega)-T(\omega)$, where $T(\omega), R(\omega)$, and $A(\omega)$ are the transmittance, reflectance, and absorption at angular frequency $\omega$, respectively. Absorption depends on the scattering parameters, for instance, $\left|S_{21}\right|^{2}=\mathrm{T}(\omega)$, and $\left|S_{11}\right|^{2}=R(\omega)$. The absorption of the incidence electromagnetic (EM) wave is simplified as $A(\omega)=1-R(\omega)$, because back side copper plane blocks the transmission of EM wave. Nonetheless, from the absorption equation, it can be seen that minimizing scattering parameters, which can maximize the absorption of the metamaterials. 


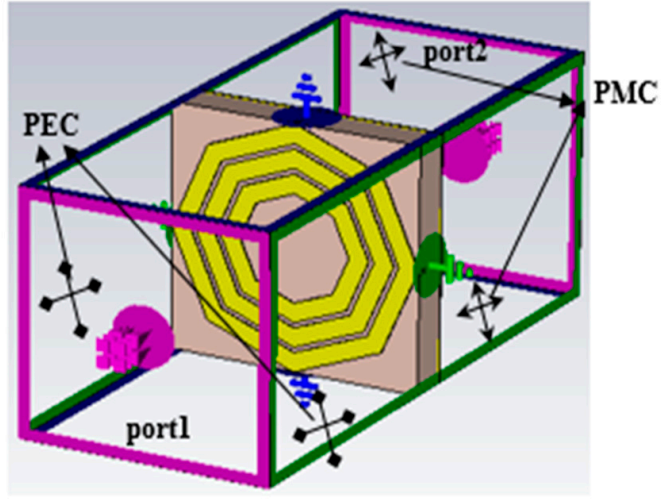

(a)

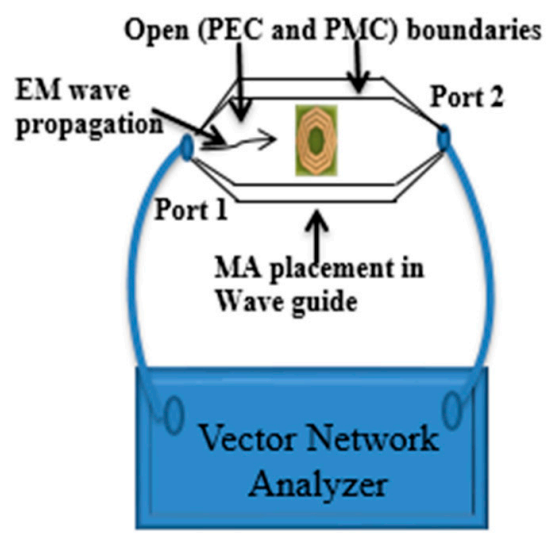

(b)

Figure 2. (a) The boundary condition of PMA; (b) Measurement set of PMA. PEC: perfect electric conductor; PMC: perfect magnetic conductor; EM: electromagnetic.

\section{Results and Discussions}

The simulations of the unit cell structure and array structure are executed using a full-wave frequency domain solver based on the FIT. In simulation, the waveguide port with periodic boundary conditions is applied. In this paper, scattering parameters, absorption of design structure with angular rotation, thickness of substrate materials, thickness of radiated patch and ground materials, effect of the resonator's number, and resistive load have been analysed. The numerical simulation and experimental result of compact octagonal shape PMA has been presented. The numerical simulation and measured absorption A $(\omega)$ of the design structure has been demonstrated in Figure 3. As is seen from Figure 3, the frequencies of the proposed design are in X-band.

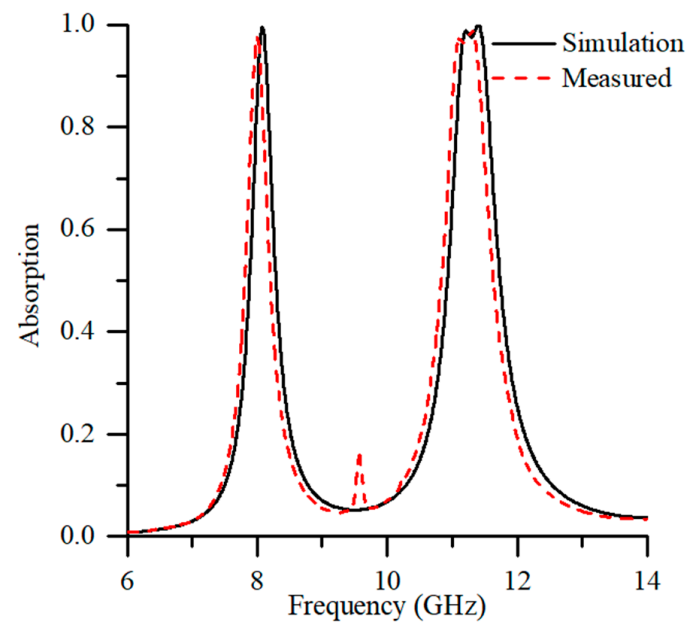

Figure 3. Simulated-measured of absorption for the proposed PMA.

From Figure 3, the numerical simulation and experimental results are showing agreement with each other. The measured results have been shown the same band when comparing with numerical results in Figure 3. In simulation, the absorption values of the structure are $99.64 \%$ and $99.95 \%$ at frequencies $8.08 \mathrm{GHz}$ and $11.41 \mathrm{GHz}$, respectively. However, the values of the absorption are $97.74 \%$ at $8.00 \mathrm{GHz}$, and $98.97 \%$ at $11.30 \mathrm{GHz}$, correspondingly by measurement. Furthermore, the absorption parameter of measured results has been slightly shifted towards the lower frequency and decrease small amount of the magnitude when comparing with simulation results. The small difference can be attributed to fabrication tolerance and open-space measurement procedure. 
The electric field and magnetic field distributions are examined at the resonant frequency of $8.08 \mathrm{GHz}$ to understand the physical mechanism of operation. The field distributions are shown in Figure 4. It is observed that there is high concentration of electric field around the outer side of the rings. The electric field are powerfully coupled with the rings and produce electric response that works as like an electric dipole moment. Hence, the charges of outer surface excite along the external electric field. As a result, a magnetic dipole is induced and it produces a magnetic response that makes a resonant absorption. In addition, high concentration of magnetic field is observed around the upper side and lower side of middle rings. The magnetic fields are strongly coupled with the rings and induce magnetic response that works as like a magnetic dipole moment. Consequently, an electric dipole is induced and produces an electric response that makes a resonant absorption. However, the desired response of electric and magnetic fields occur at this resonant frequency concurrently, resulting in almost complete absorption of the EM wave under the ideal condition $\left(Z(\omega)=Z_{0}(\omega)\right)$.

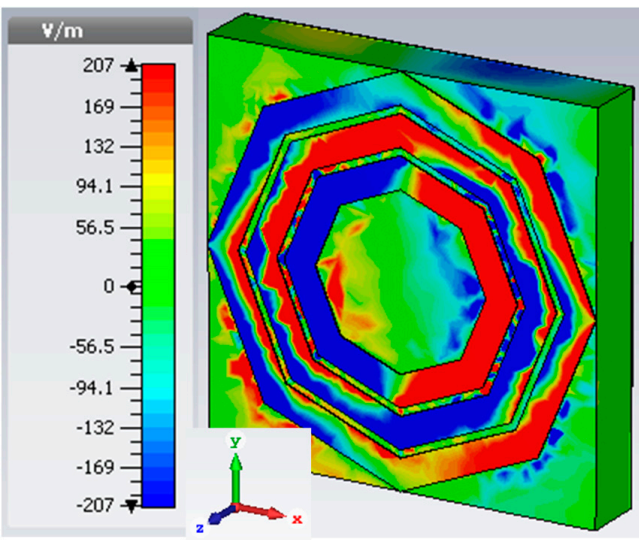

(a)

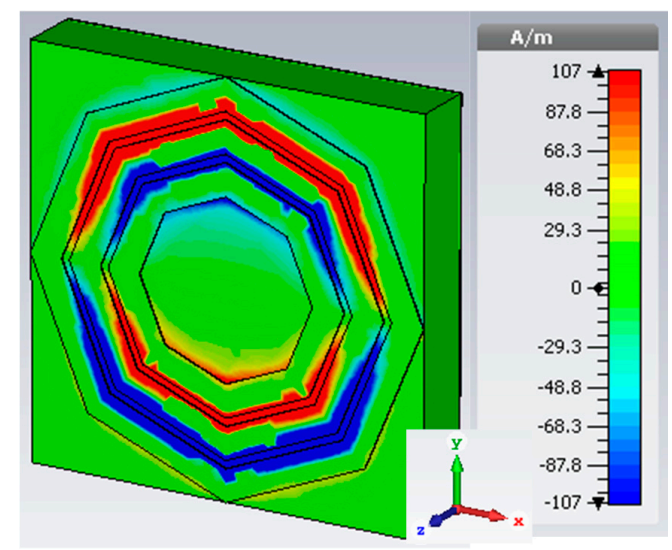

(b)

Figure 4. (a) Electric field; (b) Magnetic field distribution at resonance frequency 8.08 GHz of PMA.

Therefore, the energy of EM is dissipated in the structure, which produces zero transmission and reflection with unity absorption. Moreover, properties are also observed for the second resonance found at $11.41 \mathrm{GHz}$ suppose its approach, as shown in Figure 5. It is seen from Figure 5, the highest concentration of electric filed occurs at the right side of the octagonal resonator. The non-uniform perturbation of field distribution is also investigated. It indicates that the response of electric and magnetic field are similar to $8.08 \mathrm{GHz}$ response with higher intensity. Hence, this approach is higher than the previous one. Hence, this produces higher absorption rate than $8.08 \mathrm{GHz}$.

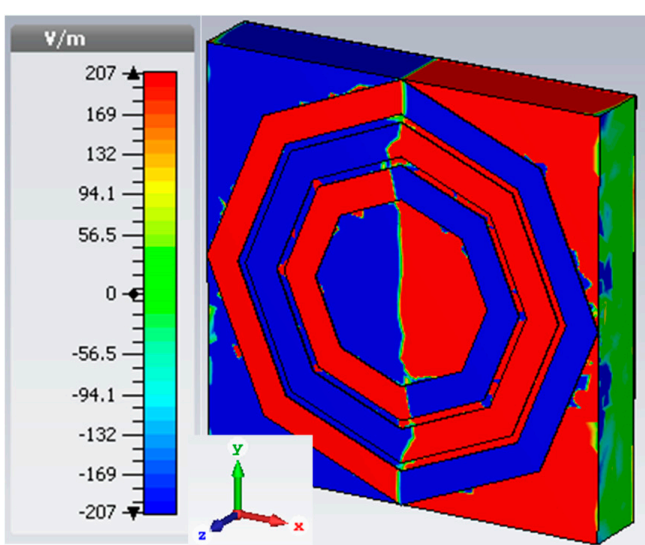

(a)

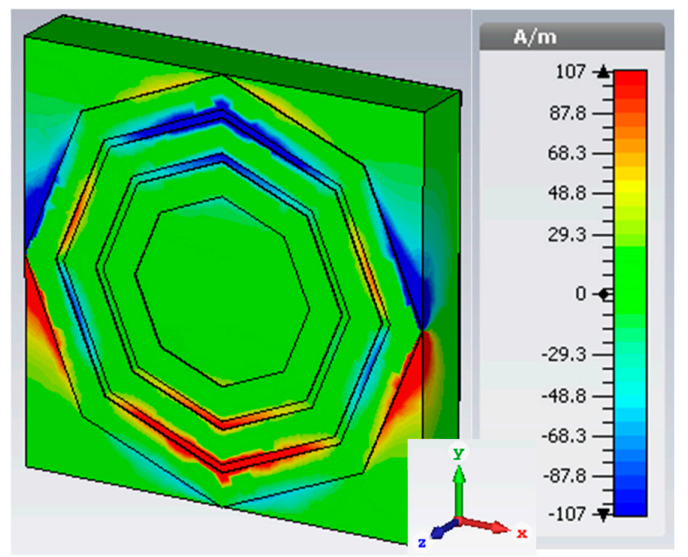

(b)

Figure 5. (a) Electric field; (b) Magnetic field distribution at resonance frequency $11.41 \mathrm{GHz}$ of PMA. 


\subsection{Analysis of Design Structure with Polarization Angle}

The numerical results of compact octagonal shape PMA have been presented. The absorption A $(\omega)$ of the design structure has been illustrated in Figure 6.

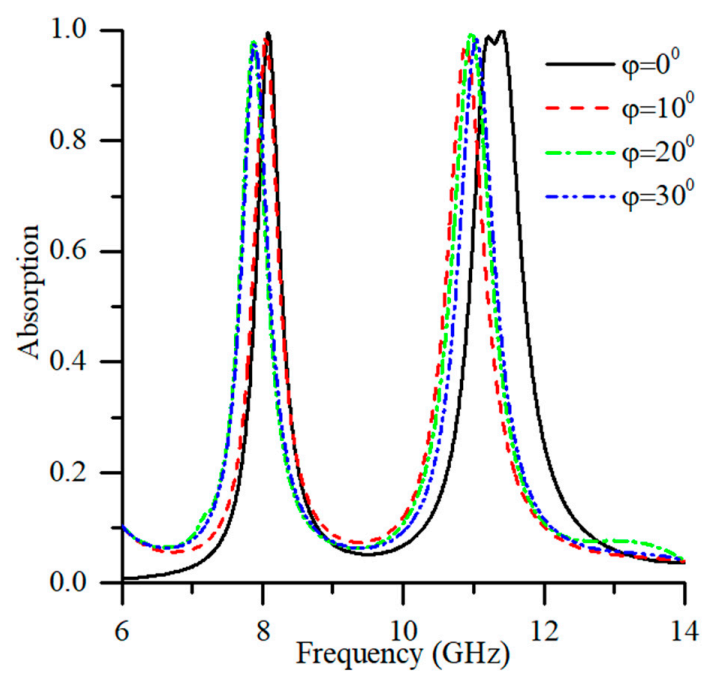

Figure 6. Absorption of design structure with polarization angle.

The effect of polarization on the design structure of PMA has been analysed and the results of the absorption is little bit changed for polarization angle. The absorption values for different polarization angle, such as $\Phi=0^{\circ}, 10^{\circ}, 20^{\circ}$, and $30^{\circ}$ are demonstrated in Figure 6. It is seen from Figure 6 that the maximum absorption values of design structure are $99.64 \%$ at $8.08 \mathrm{GHz}$, and $99.95 \%$ at $11.41 \mathrm{GHz}$, for $0^{\circ}$ polarization; the highest peaks of the absorption are $98.93 \%$ at $8.05 \mathrm{GHz}$, and $97.21 \%$ at $10.89 \mathrm{GHz}$, for $10^{\circ}$; the maximum absorption values of structure are $98.22 \%$ at $7.87 \mathrm{GHz}$, and $99.47 \%$ at $10.97 \mathrm{GHz}$, for $20^{\circ}$; and, the highest peaks of the absorption are $97.54 \%$ at $7.89 \mathrm{GHz}$, and $98.28 \%$ at $11.04 \mathrm{GHz}$, for $30^{\circ}$, respectively. At $0^{\circ}$ polarization, the design structure has exhibited highest peak of the absorption. The absorption value is high while the transmittance value is near zero. However, the reflectance value is also less at that point. The polarization of electromagnetic wave has marginally changed the resonance frequencies and absorption values due to altering the material properties.

\subsection{Analysis of Design Structure with Different Thickness of FR4 Substrate Materials}

Different thickness of substrate materials, for example, $0.8 \mathrm{~mm}, 1.6 \mathrm{~mm}$, and $2.4 \mathrm{~mm}$ have been considered in the design structure. To observe the absorption, three different thicknesses have been utilized for proposed design. It is observed from Figure 7, the absorption values of the structure are $89.20 \%$ at $7.85 \mathrm{GHz}$, and $95.76 \%$ at $10.94 \mathrm{GHz}$, respectively, for FR4 substrate material with $0.8 \mathrm{~mm}$ thick. Similarly, the values of the absorption are $99.64 \%$ at $8.08 \mathrm{GHz}$, and $99.97 \%$ at $11.40 \mathrm{GHz}$, correspondingly for $1.6 \mathrm{~mm}$ thickness. Besides, absorption values are $92.88 \%$ at $8.07 \mathrm{GHz}$ and $99.56 \%$ at $11.57 \mathrm{GHz}$, individually for $2.4 \mathrm{~mm}$ thickness of substrate material. The high absorption value are $99.64 \%$ at $8.08 \mathrm{GHz}$, and $99.97 \%$ at $11.40 \mathrm{GHz}$ for $1.6 \mathrm{~mm}$ thickness of substrate material. Due to different thickness of substrate material, the absorption rate has been different at different frequencies.

The thickness of substrate material with $1.6 \mathrm{~mm}$ has revealed the maximum peak. On the other hand, the minimum peak has been achieved from FR4 substrate material with $2.4 \mathrm{~mm}$ thickness. 


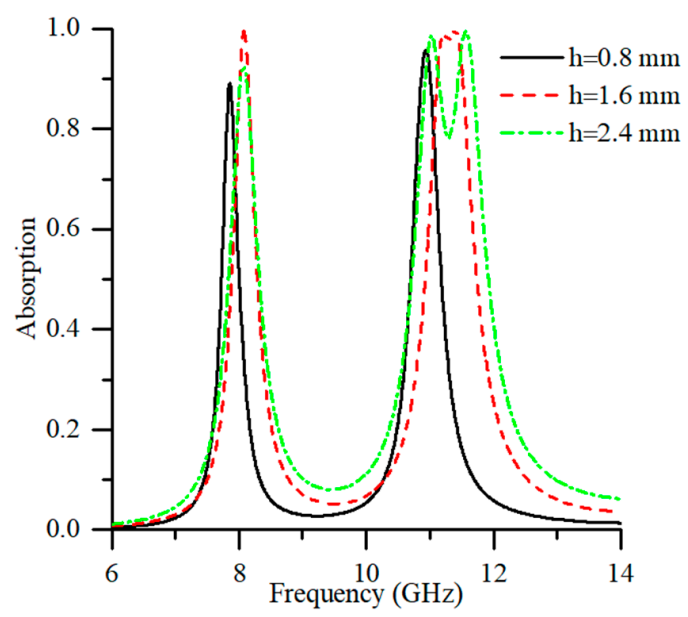

Figure 7. Absorption of the design structure for different thickness of substrate materials.

\subsection{Analysis of Design Structure with Different Thickness of Metallic Materials}

Thickness of the resonator and ground plane of the PMA has an effect on the absorption of electromagnetic energy. Different thickness like $0.1 \mathrm{~mm}, 0.2 \mathrm{~mm}, 0.3 \mathrm{~mm}$, and $0.4 \mathrm{~mm}$ have been analyzed in the design structure.

The variation of absorption is shown in Figure 8. The absorption values of the structure are $99.04 \%$ at $8.32 \mathrm{GHz}$ and $96.98 \%$ at $11.59 \mathrm{GHz}$, respectively, for metallic material with $0.1 \mathrm{~mm}$ thickness. Likewise, the values of the absorption are $97.96 \%$ at $8.66 \mathrm{GHz}$, and $97.39 \%$ at $11.89 \mathrm{GHz}$, correspondingly for $0.2 \mathrm{~mm}$ thickness. In addition, absorption values are $96.70 \%$ at $9.01 \mathrm{GHz}$ and $99.71 \%$ at $12.22 \mathrm{GHz}$, respectively, for $0.3 \mathrm{~mm}$ thickness as well as $99.04 \%$ at $9.11 \mathrm{GHz}$ and $99.92 \%$ at $12.43 \mathrm{GHz}$, respectively, for $0.4 \mathrm{~mm}$ thickness of copper metallic material. The highest absorption peaks are $99.04 \%$ at $9.11 \mathrm{GHz}$ and $99.92 \%$ at $12.43 \mathrm{GHz}$ for $0.3 \mathrm{~mm}$ thickness of metallic material. Due to different thickness of metallic material, the amount of absorption has been different at different frequencies. The thickness of metallic material with $0.3 \mathrm{~mm}$ has shown the maximum peak. However, the minimum peak has been achieved from metallic material with $0.2 \mathrm{~mm}$ thick. However, the variation of absorption has occurred in a small amount.

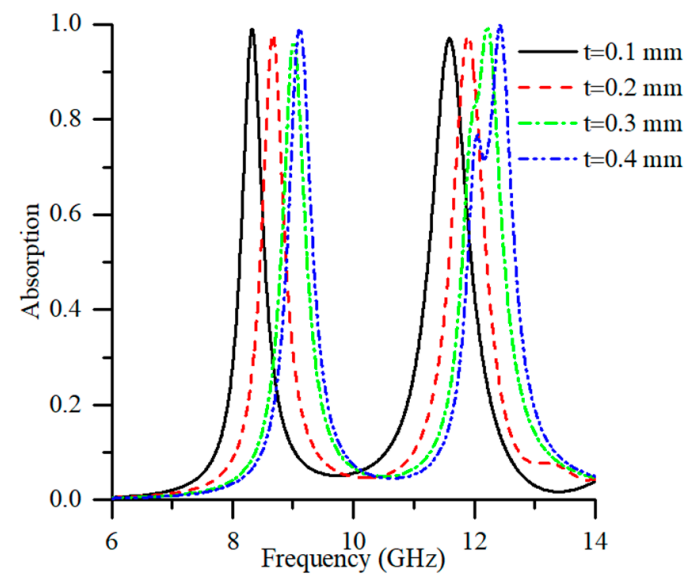

Figure 8. Absorption of the design structure for different thickness of metallic materials.

\subsection{Analysis of Design Structure with Resistive Load}

The effects of the resistive load of the octagonal resonators on the absorption have been analysed. The absorption of design structure with different values of a resistive load are shown in Figure 9. The maximum absorption peaks of the structure are $99.40 \%$ at $8.17 \mathrm{GHz}$ and $99.74 \%$ at $11.23 \mathrm{GHz}$, respectively, for $1500 \Omega$ resistive load. Similarly, the maximum peak values of the absorption are 
$99.54 \%$ at $8.19 \mathrm{GHz}$, and $99.77 \%$ at $11.41 \mathrm{GHz}$, correspondingly for $3000 \Omega$ resistive load. Additionally, absorption peak values are $99.41 \%$ at $8.20 \mathrm{GHz}$ and $99.76 \%$ at $11.26 \mathrm{GHz}$, respectively, for $4500 \Omega$ resistive load, as well as $99.33 \%$ at $8.21 \mathrm{GHz}$ and $99.94 \%$ at $11.26 \mathrm{GHz}$, respectively, for $6000 \Omega$ resistive load that placed between two rings. The high absorption peaks are $99.54 \%$ at $8.19 \mathrm{GHz}$, and $99.77 \%$ at $11.41 \mathrm{GHz}$ for $3000 \Omega$ resistive load.

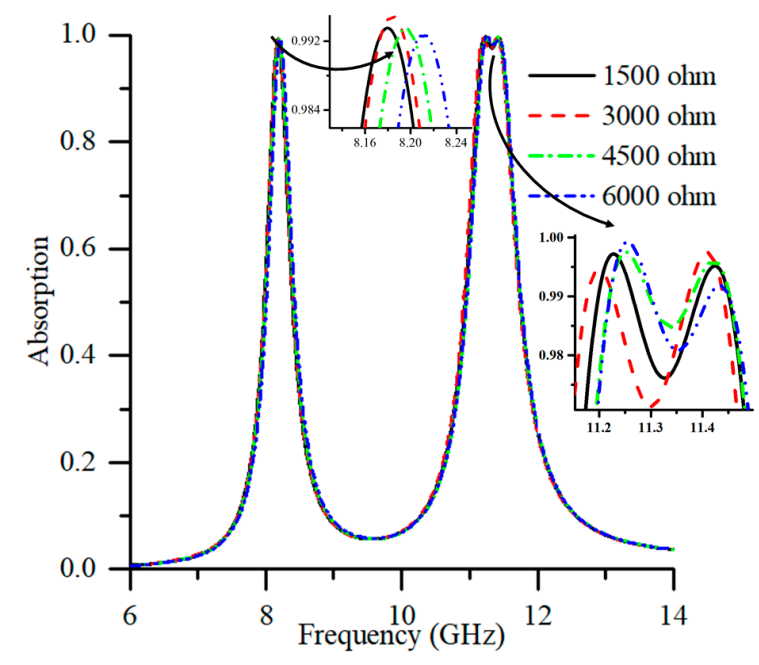

Figure 9. Absorption of the design structure for different resistive load.

Due to different resistive load, the absorption values have been different at different frequencies. The resistive load with $3000 \Omega$ has shown the maximum absorption value. On the other hand, the minimum value of absorption has been attained from resistive load with $1500 \Omega$. The effects of resistive load also showed that the performance of absorption of PMA also depends on lumped elements. Furthermore, the absorbing performance has been optimized by proper choice of the lumped resistor of the structure.

\subsection{Analysis of Design Structure with Resonator's Number}

The absorption of electromagnetic energy also is contingent on the resonator's number used in the structure. Effect of resonator's number on the substrate material of the PMA has been analyzed. The effect of absorption is shown in Figure 10. The absorption peaks of the structure are $58.35 \%$ at $7.54 \mathrm{GHz}$ and $91.97 \%$ at $10.89 \mathrm{GHz}$ for two inner resonators. Similarly, the peaks of the absorption are $81.77 \%$ at $10.99 \mathrm{GHz}$ for inner and outer resonators. Moreover, absorption peaks are $98.99 \%$ at $8.10 \mathrm{GHz}$ and $68.97 \%$ at $11.43 \mathrm{GHz}$, respectively, for two outer resonators. Furthermore, $99.64 \%$ at $8.08 \mathrm{GHz}$ and $99.95 \%$ at $11.41 \mathrm{GHz}$, respectively, for three metallic resonators. The maximum absorption peaks are $99.64 \%$ at $8.08 \mathrm{GHz}$ and $99.95 \%$ at $11.41 \mathrm{GHz}$, for three metallic resonators.

The absorption values have been different due to the number of metallic resonators. The PMA with three metallic resonators has shown the maximum peak. However, the minimum peak and single absorption peak have been achieved from metallic resonator with the combination of inner and outer resonators. Hence, the variation of absorption has occurred for different ring's number.

Table 1 demonstrates the comparisons with proposed PMA and another reported PMA. The compared parameters of PMA have been considered here, for example, design structure, size of unit cell structure, applicable band, absorption rate, and year published. Kollatou et al. [21] proposed modified square-shape structure with $8 \times 8 \mathrm{~mm}^{2}$ size and obtained absorption of $95.81 \%$ in Table 1. Likewise, other authors, Rana et al. in [22], suggested a U-shape absorber for multiband application. On the other hand, authors have achieved $98 \%$ absorption with $15 \times 15 \mathrm{~mm}^{2}$ size of design structure. Borah et al. [23] exhibited an O-shape with $12 \times 12 \mathrm{~mm}^{2}$ dimension of unit cell structure for X-band application. However, Authors obtained absorption of $98.90 \%$. In [17] an S-shape 
metamaterial absorber was analysed using various substrate materials and different propagation axis of electromagnetic wave. In contrast, authors have obtained a small amount of absorption. Sen et al. recommended L-shape structure with $9 \times 9 \mathrm{~mm}^{2}$ dimension for $\mathrm{X}$ - and $\mathrm{Ku}$-band operations and attained absorption of $95 \%$. In addition, the authors, Mahmood et al. [24] suggested modified S-shape structure with $16 \times 16 \mathrm{~mm}^{2}$ dimensions and achieved absorption of $90 \%$. The compact octagonal shape PMA has been analysed and attained higher absorption (98.97\%) with compact size of unit cell $\left(10 \times 10 \mathrm{~mm}^{2}\right)$ in this paper. The proposed metamaterial has attained compactness and high absorption comparing mentioned references that are suitable for the microwave regime. In addition, the compact size of the design structure is feasible for $\mathrm{X}$ band application. Hence, the manufacturability and robustness of the properties of our design structure are very good for commercial adoption.

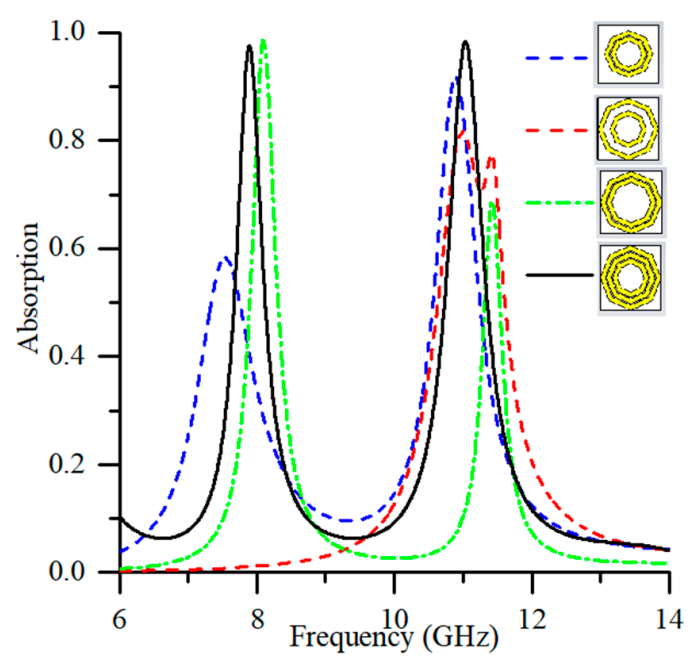

Figure 10. Absorption of the design structure for number of metallic resonators.

Table 1. Justification of previous perfect metamaterial absorber (PMA) and proposed PMA.

\begin{tabular}{cccccc}
\hline Author Name & Design Structure & Size $\left(\mathbf{m m}^{\mathbf{2}}\right)$ & Proposed Band & Absorption Rate (\%) & Year of Published \\
\hline Kollatou et al. [21] & Modified Square & $8 \times 8$ & X-band & 95.81 & 2013 \\
Rana et al. [22] & U-shape & $15 \times 15$ & X-band & 98.00 & 2016 \\
Borah et al. [23] & O-shape & $12 \times 12$ & X-band & 98.90 & 2016 \\
Islam et al. [17] & S-shape & $20 \times 20$ & S-, X-, Ku-band & 55.00 & 2017 \\
Sen et al. [25] & L-shape & $9 \times 9$ & X-, Ku-band & 95.00 & 2017 \\
Mahmood et al. [24] & Modified S-shape & $16 \times 16$ & X-band & 90.00 & 2017 \\
Proposed PMA & Octagonal shape & $10 \times 10$ & X-band & 98.97 & - \\
\hline
\end{tabular}

\section{Conclusions}

A new design of compact octagonal shape PMA was proposed for analysing the absorbing properties based on the numerical simulation and experimental results. The geometry of the offered PMA structure was very simple and it showed a high absorption in microwave frequencies. The results of measurement are in agreement with the numerical ones. The suggested PMA is appropriate for $\mathrm{x}$-band microwave application. A comparative analysis also carried out on the basis of the polarization angle of the unit cell, different thickness of substrate and metallic material, number of resonator, and energy absorption with resistive load. The mentioned variation of PMA shows good performance and the values of absorption are around unity. The open-space measurement method was applied to validate the results of the prototype of the structure. The metamaterial structure was compact in size, and high absorption, which makes it ideal for defence and stealth systems.

Acknowledgments: This work was supported by the Ministry of Education (MOE) under Fundamental Research Grant Scheme, Code: FRGS/1/2017/TK04/UKM/02/3. 
Author Contributions: Mohammad Jakir Hossain made substantial contributions to the conception, design and analysis. Mohammad Rashed Iqbal Faruque participated in revising the article critically for important intellectual contents. Mohammad Tariqul Islam and Kamarulzaman bin Mat provided necessary instructions for experimental purposes.

Conflicts of Interest: The authors declare no conflict of interest.

\section{References}

1. Smith, D.R.; Pendry, J.B.; Wiltshire, M.C. Metamaterials and negative refractive index. Science 2004, 305, 788-792. [CrossRef] [PubMed]

2. Soukoulis, C.M.; Linden, S.; Wegener, M. Negative refractive index at optical wavelengths. Science 2007, 315, 47-49. [CrossRef] [PubMed]

3. Landy, N.I.; Sajuyigbe, S.; Mock, J.; Smith, D.; Padilla, W. Perfect metamaterial absorber. Phys. Rev. Lett. 2008, 100, 207402. [CrossRef] [PubMed]

4. Singh, P.; Ameri, S.K.; Chao, L.; Afsar, M.N.; Sonkusale, S. Broadband millimeterwave metamaterial absorber based on embedding of dual resonators. Prog. Electromagn. Res. 2013, 142, 625-638. [CrossRef]

5. Huang, X.; Yang, H.; Yu, S.; Wang, J.; Li, M.; Ye, Q. Triple-band polarization-insensitive wide-angle ultra-thin planar spiral metamaterial absorber. J. Appl. Phys. 2013, 113, 213516. [CrossRef]

6. Xu, Y.Q.; Zhou, P.H.; Zhang, H.B.; Chen, L.; Deng, L.J. A wide-angle planar metamaterial absorber based on split ring resonator coupling. J. Appl. Phys. 2011, 110, 044102.

7. Shrekenhamer, D.; Xu, W.; Venkatesh, S.; Schurig, D.; Sonkusale, S.; Padilla, W.J. Experimental realization of a metamaterial detector focal plane array. Phys. Rev. Lett. 2012, 109, 177401. [CrossRef] [PubMed]

8. Shrekenhamer, D.; Watts, C.M.; Montoya, J.; Krishna, S.; Padilla, W.J. Metamaterial-based imaging for potential security applications. SPIE OPTO 2013,8632, 863221.

9. Xiong, H.; Hong, J.-S.; Luo, C.-M.; Zhong, L.-L. An ultrathin and broadband metamaterial absorber using multi-layer structures. J. Appl. Phys. 2013, 114, 064109. [CrossRef]

10. Hunt, J.; Driscoll, T.; Mrozack, A.; Lipworth, G.; Reynolds, M.; Brady, D.; Smith, D.R. Metamaterial apertures for computational imaging. Science 2013, 339, 310-313. [CrossRef] [PubMed]

11. Pfeiffer, C.; Grbic, A. Metamaterial Huygens' surfaces: Tailoring wave fronts with reflectionless sheets. Phys. Rev. Lett. 2013, 110, 197401. [CrossRef] [PubMed]

12. Naser-Moghadasi, M.; Nia, A.Z.; Toolabi, M.; Heydari, S. Microwave metamaterial Absorber based on Jerusalem Cross with meandered load for bandwidth enhancement. Opt. Int. J. Light Electron Opt. 2017, 140, 515-522. [CrossRef]

13. Lin, B.; Zhao, S.; Da, X.; Fang, Y.; Ma, J.; Li, W.; Zhu, Z. Triple-band low frequency ultra-compact metamaterial absorber. J. Appl. Phys. 2015, 117, 184503. [CrossRef]

14. Zhao, J.; Cheng, Y. Ultrabroadband microwave metamaterial absorber based on electric SRR loaded with lumped resistors. J. Electron. Mater. 2016, 45, 5033-5039. [CrossRef]

15. Dincer, F.; Karaaslan, M.; Sabah, C. Design and analysis of perfect metamaterial absorber in $\mathrm{GHz}$ and $\mathrm{THz}$ frequencies. J. Electromagn. Waves Appl. 2015, 29, 2492-2500. [CrossRef]

16. Hossain, M.J.; Faruque, M.R.I.; Islam, M.T. Design and analysis of a new composite double negative metamaterial for multi-band communication. Curr. Appl. Phys. 2017, 17, 931-939. [CrossRef]

17. Islam, S.S.; Faruque, M.R.I.; Islam, M.T. Design and absorption analysis of a new multiband split-S-shaped metamaterial. Sci. Eng. Compos. Mater. 2017, 24, 139-148. [CrossRef]

18. Kim, Y.; Yoo, Y.; Hwang, J.; Lee, Y. Ultra-broadband microwave metamaterial absorber based on resistive sheets. J. Opt. 2016, 19, 015103. [CrossRef]

19. Wen, Q.-Y.; Zhang, H.-W.; Xie, Y.-S.; Yang, Q.-H.; Liu, Y.-L. Dual band terahertz metamaterial absorber: Design, fabrication, and characterization. Appl. Phys. Lett. 2009, 95, 241111. [CrossRef]

20. Tao, H.; Bingham, C.; Pilon, D.; Fan, K.; Strikwerda, A.; Shrekenhamer, D.; Padilla, W.J.; Zhang, X.; Averitt, R.D. A dual band terahertz metamaterial absorber. J. Phys. D Appl. Phys. 2010, 43, 225102. [CrossRef]

21. Kollatou, T.M.; Dimitriadis, A.I.; Assimonis, S.; Kantartzis, N.V.; Antonopoulos, C.S. A family of ultra-thin, polarization-insensitive, multi-band, highly absorbing metamaterial structures. Prog. Electromagn. Res. 2013, 136, 579-594. [CrossRef] 
22. Rana, C.R.; Inamdar, K.; Lalluwadia, A. Study, Design and Analysis of U-Shaped Metamaterial Absorber for X-Band Application. IUP J. Telecommun. 2016, 8, 23.

23. Borah, D.; Bhattacharyya, N.S. Design and Development of Expanded Graphite-Based Non-metallic and Flexible Metamaterial Absorber for X-band Applications. J. Electron. Mater. 2017, 46, 226-232. [CrossRef]

24. Mahmood, A.; Yetkin, G.Ö.; Sabah, C. Design and Fabrication of a Novel Wideband DNG Metamaterial with the Absorber Application in Microwave X-Band. Adv. Condens. Matter Phys. 2017, 2017, 1279849. [CrossRef]

25. Sen, G.; Islam, S.N.; Banerjee, A.; Das, S. Broadband Perfect Metamaterial Absorber on Thin Substrate for X-Band and Ku-Band Applications. Prog. Electromagn. Res. C 2017, 73, 9-16. [CrossRef]

(C) 2017 by the authors. Licensee MDPI, Basel, Switzerland. This article is an open access article distributed under the terms and conditions of the Creative Commons Attribution (CC BY) license (http:/ / creativecommons.org/licenses/by/4.0/). 